\title{
Dark galactic halos without dark matter
}

\author{
R. K. NEsBET \\ IBM Almaden Research Center, 650 Harry Road, San Jose, CA 95120-6099, USA
}

PACS 04.20.Cv - Fundamental problems and general formalism

PACS 98.80.-k - Cosmology

PACS 98.62.Gq - Galactic halos

\begin{abstract}
Using standard Einstein theory, baryonic mass cannot account for observed galactic rotation velocities and gravitational lensing, attributed to galactic dark matter halos. In contrast, theory constrained by Weyl conformal scaling symmetry explains observed galactic rotation in the halo region without invoking dark matter. An explanation of dark halos, gravitational lensing, and structural stabilization, without dark matter and consistent with conformal theory, is proposed here. Condensation of uniform primordial matter into a material cloud or galaxy vacates a large surrounding spherical halo. Within such an extended vacancy in the original cosmic background mass-energy density, conformal theory predicts centripetal acceleration of the observed magnitude.
\end{abstract}

Introduction. - The concept of dark matter galactic halos originates from dynamical studies [1,2] which indicate that a spiral galaxy such as our own would lack ' long-term stability if not supported by an additional gravitational field from some unseen source. This concept is supported by other cosmological data [3, including gravitational lensing [4,5. Excess centripetal acceleration ob'served in orbital velocities and lensing is attributed in standard $\Lambda \mathrm{CDM}$ theory [3, 6, 7, to a dark matter halo. ' A central conclusion of $\Lambda \mathrm{CDM}$ cosmology is that inferred dark matter outweighs baryonic matter.

In the $\Lambda$ CDM model, an isolated galaxy is considered to be surrounded by a much larger spherical dark mat' ter halo. What is actually observed is a halo of gravitational field that deflects photons in gravitational lensing and increases the velocity of orbiting mass particles. It will be shown here that conformal theory [8] 10, which modifies both Einstein-Hilbert general relativity and the Higgs scalar field model, supports an alternative interpretation of lensing and anomalous rotation as gravitational effects due to depletion of the cosmic background by concentration of diffuse primordial mass into an observed galaxy.

In standard theory, Poisson's equation determines a distribution of dark matter for any unexplained gravitational field. If dark matter interacts only through gravity, the concept of dark matter provides a compact description of observed phenomena, not a falsifiable explanation. Postulated universal conformal symmetry [10] promises a falsifiable alternative, not requiring any novel elementary fields.

Conformal gravity theory is based on the conformally invariant Weyl tensor [11. Possible formal difficulties 1214 have been discussed and resolved in detail [15]17. Conformal Higgs theory is a uniquely defined version of standard $\phi^{4}$ field theory [8, 18].

As a galaxy forms, background matter density $\rho_{m}$ condenses into observed galactic density $\rho_{g}$. Conservation of mass and energy requires total galactic mass $M$ to be missing from a depleted background. Since the primordial density is uniform and isotropic, the depleted background can be modeled by an empty sphere of radius $r_{H}$, such that $4 \pi \rho_{m} r_{H}^{3} / 3=M$. In particular, the integral of $\rho_{g}-\rho_{m}$ must vanish. The resulting gravitational effect, derived here from conformal theory, describes a dark halo. This halo model accounts for the otherwise remarkable fact that galaxies of all shapes are embedded in essentially spherical halos. Conformal theory relates the radial acceleration parameter of anomalous galactic rotation to the observed acceleration of Hubble expansion.

Assuming that galactic mass is concentrated within an average radius $r_{g}$, the ratio of radii $r_{H} / r_{g}$ should be very large, the cube root of the mass-density ratio $\rho_{g} / \rho_{m}$. Thus if the latter ratio is $10^{5}$ a galaxy of radius $10 \mathrm{kpc}$ would be accompanied by a halo of radius $10 \times 10^{5 / 3}=464 \mathrm{kpc}$. Equivalence of galactic and displaced halo mass resolves the paradox for $\Lambda \mathrm{CDM}$ that despite any interaction other than gravity, the amount of dark matter inferred for a galactic halo is strongly correlated with the galactic luminosity or baryonic mass [7, 19.

Together with conformal theory of anomalous galactic rotation velocities [8, 9, and Hubble expansion [18, the 
present halo model removes any need to invoke dark matter for major isolated galactic phenomena [10. This suggests that when applied to other cosmological phenomena, galactic growth and interaction and the original bigbang itself, conformal theory will provide an alternative paradigm to $\Lambda$ CDM. It is shown here that the NewtonEinstein model of galactic growth and interaction is substantially altered by conformal theory.

Gravitational effect of a depleted halo. - What, if any, would be the gravitational effect of a depleted background density? An analogy, in well-known physics, is impurity scattering of electrons in conductors. In a complex material with a regular periodic lattice, independent electron waves are by no means trivial functions, but they propagate without contributing to scattering or resistivity unless there is some lattice irregularity, such as a vacancy or substituted atom. Impurity scattering depends on the difference between impurity and host atomic T-matrices 20. Similarly, a photon or isolated mass particle follows a geodesic in the cosmic background unless there is some disturbance of uniform background density $\rho_{m}$.

A gravitational halo is treated here as a natural consequence of galaxy formation. Condensation of matter from the primordial uniform mass-energy distribution leaves a depleted sphere that has an explicit gravitational effect. The implied subtracted mass, which integrates to minus the total galactic mass, cannot be ignored. If this mass were simply removed, the analogy to vacancy scattering in solids implies a lensing effect. A background geodesic is no longer a geodesic in the empty sphere.

Following the basic concepts of general relativity, deflection of a geodesic would be observed as radial acceleration of orbiting mass particles. It will be shown here that conformal theory [8,10,18, explains the observed centripetal character of halo gravitation, without invoking dark matter. In standard theory, subtracted density $-\rho_{m}$ would predict centrifugal acceleration, contrary to observation.

Analyses of galactic rotation velocities using conformal gravity [8,21] 24, the MOND model 25],26, or the scalarvector-tensor theory of Moffat 27,28, all without dark matter, fit observed data. Analysis of Hubble expansion using the conformal Higgs scalar field model [18,29] agrees with consensus cosmological data 30 back to the CMB epoch, also without assuming dark matter.

The fit of conformal gravity to anomalous galactic rotation data implies a significant effect of the cosmic background, external to a baryonic galactic core $[8,21,32$. The inferred centripetal radial acceleration, independent of galactic structure and mass, is attributed here to a galactic halo depleted of its original mass density. This is consistent with conformal theory of both galactic rotation and Hubble expansion 10. Observed centrifugal acceleration of Hubble expansion 33, 34 is verified by the conformal Higgs model [18. As will be shown here, the change of Hubble acceleration due to depleted background mass density is consistent with the observed centripetal acceleration due to a gravitational halo.

Summary of relevant theory. - Variational theory for fields in general relativity is a straightforward generalization of classical field theory $[8,35,36$. Given Riemannian scalar Lagrangian density $\mathcal{L}$, action integral $I=\int d^{4} x \sqrt{-g} \mathcal{L}$ is required to be stationary for all differentiable field variations, subject to appropriate boundary conditions. $g$ here is the determinant of metric tensor $g_{\mu \nu}$. Gravitational field equations are determined by metric functional derivative $X^{\mu \nu}=\frac{1}{\sqrt{-g}} \frac{\delta I}{\delta g_{\mu \nu}}$. Any scalar $\mathcal{L}_{a}$ determines energy-momentum tensor $\Theta_{a}^{\mu \nu}=-2 X_{a}^{\mu \nu}$, evaluated for a solution of the field equations.

Strict conformal symmetry 8, 11 requires invariance of field action integrals under local Weyl scaling, such that $g_{\mu \nu}(x) \rightarrow g_{\mu \nu}(x) \alpha^{2}(x)$, where $\alpha(x)$ is real and differentiable. For a scalar field, $\Phi(x) \rightarrow \Phi(x) \alpha^{-1}(x)$. A conformal energy-momentum tensor must be traceless. This is true for massless fermion and gauge boson fields, but not for Einstein tensor $G^{\mu \nu}$. Conformal theory [8,10, which determines unique Lagrangian densities $\mathcal{L}_{g}$ for the metric tensor and $\mathcal{L}_{\Phi}$ for a scalar field, removes this inconsistency. Subgalactic phenomenology is preserved, but gravitation on a galactic scale is modified 32. The conformal Higgs model determines dark energy [18,29.

The conformal gravitational field equation is

$$
X_{g}^{\mu \nu}+X_{\Phi}^{\mu \nu}=\frac{1}{2} \Theta_{m}^{\mu \nu},
$$

where index $\mathrm{m}$ refers to matter and radiation. An exact solution inside the halo radius is given by

$$
X_{g}^{\mu \nu}=\frac{1}{2} \Theta_{m}^{\mu \nu}\left(\rho_{g}\right), X_{\Phi}^{\mu \nu}=0, r \leq r_{H} .
$$

The exact source-free solution 32 of the $X_{g}$ equation is valid in the external halo, $r_{g} \leq r \leq r_{H}$, because $\rho_{g}$ vanishes. Constants of integration fitted at $r_{g}$ and $r_{H}$ determine radial acceleration in this external halo. $X_{\Phi}^{\mu \nu}=0$ is solved exactly using the modified Friedmann equation [18 with mass-energy density omitted.

Given any uniform mass-energy density $\rho$, for $r \leq r_{H}$, field equation $X_{\Phi}^{\mu \nu}(\rho)=\frac{1}{2} \Theta_{m}^{\mu \nu}(\rho)$ implies a modified Friedmann equation [18. Solution for scale factor $a(t)$ determines dimensionless Friedmann acceleration weight $\Omega_{q}(\rho)=\frac{\ddot{a} a}{\dot{a}^{2}}$ [18. For uniform $\rho=0,0 \leq r \leq r_{H}$, this solves the second of Eqs.(2) within the halo radius. However, the $X_{\Phi}$ equation includes dark energy, present regardless of density $\rho$. This produces the centrifugal acceleration of background Hubble expansion [10, 18, which must be subtracted off in order to compute observable radial acceleration. Observed geodesics, whose bending determines the extragalactic centripetal acceleration responsible for lensing and orbital rotation velocities, are defined relative to the cosmic background.

What is proposed here is to solve modified Friedmann equations for both $X_{\Phi}^{\mu \nu}\left(\rho_{m}\right)$ and $X_{\Phi}^{\mu \nu}(0)$ to obtain Friedmann acceleration weights at present time $t_{0}, \Omega_{q}\left(\rho_{m}\right)$ and 
$\Omega_{q}(0)$ respectively. The observed radial acceleration is $\Delta \Omega_{q}=\Omega_{q}(0)-\Omega_{q}\left(\rho_{m}\right)$, which cancels acceleration due to dark energy, much greater at present time than that due to baryonic mass. This implements the argument given above, that observed effects are determined by subtracting out the uniform background mass-energy density. The gravitational field due to $\rho_{g}$ is augmented by a halo field due to $-\rho_{m}$.

The gravitational field within a depleted halo. -

Analysis here is greatly simplified by solving the field equations in two different metric systems, made compatible by choice of constants of integration. The exterior Schwarzschild (ES) metric is valid for $X_{g}^{\mu \nu}$ and galactic rotational velocities in the external halo 8 , while the Robertson-Walker (RW) metric is valid for $X_{\Phi}^{\mu \nu}$ and Hubble expansion [18.

In conformal theory the most general metric outside a static spherically symmetric source density takes the exterior Schwarzschild form 32

$$
d s_{E S}^{2}=-B(r) d t^{2}+\frac{d r^{2}}{B(r)}+r^{2} d \omega^{2}
$$

Here $c=\hbar=1$ and $d \omega^{2}=d \theta^{2}+\sin ^{2} \theta d \phi^{2}$. Because the Weyl tensor vanishes identically for the assumed uniform, isotropic void outside a spherical baryonic galactic core, conformal $X_{g}^{\mu \nu}=0$ in the external halo. An exact solution of this source-free field equation outside source radius $r_{g}$ is given in the ES metric by potential function 32

$$
B(r)=1-2 \beta / r+\gamma r-\kappa r^{2}, r \geq r_{g} .
$$

A circular orbit outside $r_{g}$ is stable for velocity such that $v^{2}=\frac{1}{2} r \frac{d B}{d r}=\beta / r+\frac{1}{2} \gamma r-\kappa r^{2}$. Centripetal radial acceleration is $v^{2} / r$. An assumed exact solution of the inhomogeneous field equation

$$
X_{g}^{\mu \nu}\left(\rho_{g}\right)=\frac{1}{2} \Theta_{m}^{\mu \nu}\left(\rho_{g}\right), r \leq r_{g},
$$

is extended out to halo radius $r_{H}$ by matching to this known external solution at $r_{g}$ [32].

In the depleted halo, integration parameters in $X_{g}^{\mu \nu}\left(\rho_{g}\right)=0$ are determined by continuity of the implied acceleration field at $r=r_{g}$ and at $r=r_{H}$, where acceleration must vanish for continuity with the unmodified external cosmos. Newtonian parameter $\beta=G M$ is proportional to total galactic mass $M$ 32. Neglecting parameter $\kappa$, Mannheim [21] determined two universal parameters such that $\gamma=\gamma^{*} N^{*}+\gamma_{0}$ fits rotational data for eleven typical galaxies, not invoking dark matter. $N^{*}$ here is total visible plus gaseous mass in units of solar mass. This has recently been extended to 138 galaxies whose orbital velocities are known outside the optical disk 22,24 . Parameter $\kappa$ was fitted as a global constant, not determined by a specific boundary condition. As discussed below, the present halo model indicates that $\kappa$ should be treated as a constant of integration whose value is determined by physical boundary conditions, in particular at the halo radius $r_{H}$.

$\gamma_{0}$ is found to have a universal value, independent of any particular galaxy. It is attributed here to the subtracted density $\rho_{m}$ of the present halo model. In the Schwarzschild metric, a solution of homogeneous equation $X_{g}^{\mu \nu}=0$ valid at the galactic center excludes the singular potential $-2 \beta_{0} / r$. Extending out to halo radius $r_{H}$, $B_{0}(r)=1+\gamma_{0} r-\kappa_{0} r^{2}$, where $\kappa_{0}=\gamma_{0} / 2 r_{H}$ terminates radial acceleration at the halo boundary. There is no interior boundary value to determine acceleration parameter $\gamma_{0}$, which must however be consistent with the modified Friedmann equation implied by $X_{\Phi}^{\mu \nu}=0$ in the uniform, isotropic Robertson-Walker metric, as shown below. Radial acceleration due to $\rho_{m}$ is determined by its differential effect on this homogeneous field equation. This follows from the fact that the mass-energy weight parameter in the modified Friedmann equation is much smaller than the dark energy weight, which determines Hubble expansion acceleration in the current epoch [18.

A uniform, isotropic cosmos with Hubble expansion is described by the Robertson-Walker (RW) metric [35.

$$
d s_{R W}^{2}=-d t^{2}+a^{2}(t)\left(\frac{d r^{2}}{1-k r^{2}}+r^{2} d \omega^{2}\right),
$$

for curvature parameter $k$. The Weyl tensor and resulting conformal Lagrangian density $\mathcal{L}_{g}$ vanish identically in this metric. Lagrangian density $\mathcal{L}_{\Phi}$ of the conformal Higgs model 18 contains Higgs tachyonic mass term $w^{2} \Phi^{\dagger} \Phi$ and $-\frac{1}{6} R \Phi^{\dagger} \Phi$, dependent on gravitational Ricci scalar $R$. The scalar field equation has an exact solution such that $\Phi^{\dagger} \Phi=\phi_{0}^{2}$, a spacetime constant if the time variation of $R$ (on a cosmological time scale) is neglected. The conformal Higgs model [18 determines a modified Friedmann cosmic evolution equation for scale parameter $a(t)$ that fits cosmological data back to the CMB epoch [30, 31] without invoking dark matter. Higgs parameter $w^{2}$ becomes dark energy [18,29] in this equation.

The modified Friedmann equation [10, 18] is

$$
\frac{\dot{a}^{2}}{a^{2}}+\frac{k}{a^{2}}-\frac{\ddot{a}}{a}=\frac{2}{3}(\bar{\tau} \rho+\bar{\Lambda}),
$$

where energy density $\rho=\Theta_{m}^{00}$. The parameters here are $\bar{\Lambda}=\frac{3}{2} w^{2}$ and $\bar{\tau}=-3 y^{2} / \phi_{0}^{2}$. Numerical factor $y^{2}$, which must be determined from empirical data, allows for a dimensionless coefficient of conformal $\mathcal{L}_{\Phi}$, nominally taken to be unity. The Newton gravitational constant is replaced by a parameter of different sign and magnitude. The Einstein tensor is replaced by a traceless conformal tensor. Vanishing total trace [8] reduces the second Friedmann equation of standard theory to an identity.

Dividing by $(\dot{a} / a)^{2}$ determines dimensionless sum rule $\Omega_{m}+\Omega_{\Lambda}+\Omega_{k}+\Omega_{q}=1$ for the modified equation. Radiation energy density is included in $\Omega_{m}$ here. The dimensionless weights are $\Omega_{m}(t)$ for mass density, $\Omega_{k}(t)$ for 
curvature, and $\Omega_{\Lambda}(t)$ for dark energy, augmented by acceleration weight $\Omega_{q}(t)=\frac{\ddot{a} a}{\dot{a}^{2}}[18$. Galactic rotation velocities, observed at relatively small redshifts, determine radial acceleration values which can be compared with acceleration weights inferred from Hubble expansion data.

Parameters $\gamma$ and $\kappa$. - Mannheim parameter $\gamma_{0}>$ 0 , independent of galactic mass and structure, implies centripetal acceleration due to an isotropic cosmological source 21]. The parametrized gravitational field forms a spherical halo 10. The depleted halo model removes a particular conceptual problem in fitting $B(r)$ parameters $\gamma, \kappa$ of $d s_{E S}^{2}$ to galactic rotation data [8, 21, 22, In empirical parameter $\gamma=\gamma^{*} N^{*}+\gamma_{0}, \gamma_{0}$ does not depend on galactic mass, so must be due to the surrounding cosmos 21. Mannheim considers this to represent the net effect of distant matter, integrated out to infinity [8]. Since the interior term, coefficient $\gamma^{*} N^{*}$, is centripetal, one might expect the term in $\gamma_{0}$ to be centrifugal, describing attraction to an exterior source. However, if coefficient $\gamma_{0}$ is due to a depleted halo, the implied sign change determines net centripetal acceleration, in agreement with observation.

Integration parameter $\kappa$, included in fitting rotation data [22 23], cuts off gravitational acceleration at a boundary radius. In the halo model, $\kappa$ is determined by the boundary condition of continuous acceleration field at halo radius $r_{H}$, determined by galactic mass, except for the nonclassical linear potential term due to to the baryonic galactic core. If this were determined directly by the $X_{g}$ equation for the material galaxy, there is no obvious reason why it should terminate at the halo boundary. In a galactic cluster, mass conservation prevents interpenetrating halos, which may determine effective radii.

Universal parameter $\gamma_{0}$ implies a halo contribution to function $v^{2}(r)$ that is the same for all galaxies, deviating only as $r$ approaches the halo radius. For galaxies of the same mass, the full parameter $\gamma=\gamma^{*} N^{*}+\gamma_{0}$ implies identical "dark matter" rotation curves, as exemplified for galaxies NGC2403 and UGC128 in Fig.1 of Reference [19]. This supports the empirical argument for a universal nonclassical rotation curve [7] and for a fundamental relationship between observed baryonic mass and inferred dark mass [19]. Such a relationship is an immediate consequence of the depleted halo model.

The halo model defines cutoff parameter $\kappa_{\text {core }}=$ $G M / r_{H}^{3}$ and nonclassical $\kappa_{0}=\gamma_{0} / 2 r_{H}$, which enforce continuity by terminating the acceleration field at $r_{H}$. For $r \leq r_{H}$ but outside baryonic density bound $r_{g}, v^{2}$ for galactic rotation is the sum of three independent terms:

$$
\begin{gathered}
v_{\text {core }}^{2}=\frac{G M}{r}\left(1-r^{3} / r_{H}^{3}\right), \\
v_{\text {halo }}^{2}=\frac{1}{2} \gamma_{0} r\left(1-r / r_{H}\right), \\
v_{\text {ncl }}^{2}=\frac{1}{2} N^{*} \gamma^{*} r\left(1-r / r_{*}\right) .
\end{gathered}
$$

Recent analysis of the $X_{g}$ field equation in the Schwarzschild metric [17] indicates that Mannheim parameter $\gamma^{*}$ is in fact determined by the $X_{\Phi}$ equation, in analogy to $\gamma_{0}$. This would imply that $\gamma^{*} r$ should also cut off at $r_{H}$. In either case, parameter $\kappa$ invalidates the Newtonian virial theorem for galactic clusters. Implications are discussed below. It would be informative to fit galactic rotation data [22,23] using $\kappa=\kappa^{*} N^{*}+\kappa_{0}$. Deviations of $\kappa$ from a universal constant might determine halo radii dependent on baryonic mass, while $\kappa^{*}$ would test whether or not $\gamma^{*} r$ is cut off at the halo radius.

Parameters $\Omega_{k}, \Omega_{m}$, and $\gamma_{0} \cdot-$ In the external halo, for $r_{g} \leq r \leq r_{H}$, a solution of $X_{g}^{\mu \nu}=0$ is determined by ES metric constants of integration $\beta, \gamma, \kappa$, proportional to galactic mass, fitted at $r_{g}$ to an interior solution for $\rho_{g} \neq 0$ [32. $X_{g}^{\mu \nu}=0,0 \leq r \leq r_{H}$ for primordial uniform background $\rho_{m}$, because the Weyl tensor vanishes. The exact background solution, regular at the coordinate origin, can only affect Mannheim parameters $\gamma$ and $\kappa$. Subtraction of the background can be expressed in terms of Friedmann acceleration weight parameters $\Omega_{q}$ in the RW metric. Geodesic deflection in the halo is due to net acceleration $\Delta \Omega_{q}=\Omega_{q}$ (halo $)-\Omega_{q}($ cosmos $)$, related to $\Delta \rho=\rho_{g}-\rho_{m}=-\rho_{m}$ within the halo. ES parameter $\gamma_{0}$ must be consistent with RW acceleration $\Delta \Omega_{q}$.

$\Delta \Omega_{q}$ can be approximated in the halo (where $X_{g}^{\mu \nu}=0$ ) by solving equation $X_{\Phi}^{\mu \nu}$ (halo) $=0$ in the RW metric to determine Friedmann weight $\Omega_{q}$ (halo), and by solving $X_{\Phi}^{\mu \nu}($ cosmos $)=\frac{1}{2} \Theta_{m}^{\mu \nu}\left(\rho_{m}\right)$ to determine $\Omega_{q}($ cosmos $)$. The modified Friedmann equation, with relevant parameters, is exact in each case. From the modified Friedmann sum rule, net acceleration weight $\Omega_{q}(\operatorname{cosmos})=1-\Omega_{\Lambda}-$ $\Omega_{k}-\Omega_{m}$. If the halo is a true vacuum, in which both $\rho_{m}$ and curvature parameter $k$ vanish, $\Omega_{q}($ halo $)=1-\Omega_{\Lambda}$, so that $\Delta \Omega_{q} \simeq \Omega_{k}+\Omega_{m}$, neglecting any change in $\Omega_{\Lambda}$.

Positive $\rho_{m}$ implies $\Omega_{m}<0$, because of negative coefficient $\bar{\tau}$. Curvature $k \geq 0$, such that $\Omega_{k}($ cosmos $) \leq 0$, implies a metric singularity at large $r$ which may be related to Hubble radius $c / H_{0}$. For nonnegative cosmic curvature, $\Delta \Omega_{q}<0$, implying centripetal acceleration and positive $\gamma_{0}$, consistent with observed anomalous galactic rotation [8, 21, 22. In Hubble units such that $\hbar=c=1$ and Hubble function $\dot{a} / a=1$, at present time $t_{0}$, for $\gamma_{0}=3.06 \times 10^{-30} / \mathrm{cm}$, deduced from galactic rotation data [8,21,22, and Hubble length unit $\frac{c}{H_{0}}=1.314 \times 10^{28} \mathrm{~cm}$, radial acceleration implied by $\gamma_{0}$ is $-\frac{1}{2} \gamma_{0} \frac{c}{H_{0}}=-0.0201$. The corresponding acceleration weight $\Delta \Omega_{q} \simeq \Omega_{k}+\Omega_{m}=-0.0201$ is consistent with empirical $\Omega_{k}=-0.0125 \pm 0.0065$ [31].

Empirical $\gamma_{0}$ may give the most accurate current estimate of $\Omega_{k}+\Omega_{m}$. Hubble expansion data for redshifts $z \leq 1$ can be fitted with $\Omega_{k}+\Omega_{m}=0$ [18. Accurate data for large redshifts and for the CMB is required for an independent value of this sum and for the individual Friedmann weights. Further analysis of the cosmic curvature parameter and of the time variation of Higgs model pa- 
rameters is needed. Consistency of lensing and anomalous rotation constrains both $\Lambda \mathrm{CDM}$ and conformal models. Accurate rotational and lensing data for the same galaxy would provide a quantitative test of theory.

Galaxy formation and galactic clusters. - A depleted halo accompanies any condensation of the cosmic background. The resulting centripetal gravitation stabilizes such condensations, so that concentration and stabilization are concurrent. It is unnecessary to assume an initial concentration of dark matter.

A new rule must be valid for galactic collisions: the total empty volume must remain equal to the total galactic mass divided by the cosmic background mass density $\rho_{m}$. Halos cannot overlap, but must distort the background density to preserve total empty volume. This may have the effect of limiting galactic growth in clusters. Two halos in contact would remove background matter that might otherwise fall into either central galaxy.

The intergalactic potentials derived here terminate either at the halo radius or at some radius to be determined by dynamic modeling. Hence the Newtonian virial theorem is not valid for clusters. Relative kinetic energy of colliding galaxies would be partially converted to thermal energy of the redistributed cosmic background density. Detailed modeling, starting with two colliding halos with their central galaxies, is needed to estimate the net thermal energy transferred to intergalactic dust or gas in a galactic cluster. The need for dark matter in galactic clusters should be reexamined on the basis of such modeling.

The rules for galaxy formation are modified. Current dynamical models of galaxy and galactic cluster formation should be revised to take into account concurrent halo formation, absence of dark matter, and modified intergalactic potentials. It would be informative to test conformal theory using a revised dynamical model. Conclusions of standard dynamical models should be reexamined.

The MOND model [3, 26], which has been applied successfully to a wide variety of cosmological phenomena, parametrizes an assumed failure of Newtonian dynamics for acceleration less than a universal constant. Similarly, conformal theory finds new gravitational effects (acceleration parameters $\gamma$ ) when Keplerian radial acceleration in a galaxy drops to a value comparable to that due to the universal effect of a galactic halo, as considered here. This suggests that other successful applications of MOND, not yet studied by conformal theory, will also turn out to be explained when such studies are carried out.

Conclusions. - Galaxy formation by condensation from the primordial cosmos implies a gravitational halo field due to depletion of the original uniform isotropic mass distribution. Standard Einstein-Hilbert theory, implying centrifugal acceleration of a photon or orbiting mass particle in a depleted halo, is contradicted by observed centripetal lensing and enhanced rotation velocities, implied by conformal theory.
Conformal gravity, the conformal Higgs model, and the present depleted halo model are mutually consistent. Together they account for observed excessive galactic rotation velocities, Hubble expansion, stabilization of growing galaxies, and galactic lensing, without invoking dark matter, and explain the source and magnitude of dark energy.

\section{REFERENCES}

[1] Ostriker J. P. and Peebles P. J. E., ApJ, 186 (1973) 467.

[2] Ostriker J. P., Peebles P. J. E. and Yahil A., ApJ, 193 (1974) L1.

[3] Sanders R. H., The Dark Matter Problem (Cambridge Univ. Press, NY) 2010.

[4] Walsh D., Carswell R. F. and Weymann R. J., $\mathrm{Na}$ ture, 279 (1979) 381.

[5] Hoekstra H. et al, ApJ, 504 (1998) 636.

[6] Dodelson S., Modern Cosmology (Academic Press, NY) 2003.

[7] Salucci P. et al, MNRAS, 378 (2007) 41.

[8] Mannheim P. D., Prog.Part.Nucl.Phys., 56 (2006) 340.

[9] Mannheim P. D., Found.Phys., 42 (2012) 388.

[10] Nesbet R. K., Entropy, 15 (2013) 162.

[11] Weyl H., Sitzungber.Preuss.Akad.Wiss., (1918) 465; Math.Zeit., 2 (1918) 384.

[12] Flanagan E. E., Phys.Rev.D, 74 (2006) 023002.

[13] Brihaye Y. and Verbin Y., Phys.Rev.D, 80 (2009) 124048.

[14] Yoon Y., Phys.Rev.D, 88 (2013) 027504.

[15] Mannheim P. D., Phys.Rev.D, 75 (2007) 124006.

[16] Mannheim P. D., Gen.Relativ.Gravit., 43 (2011) 703.

[17] Nesbet R. K., arXiv:1410.8076v2, (2014).

[18] Nesbet R. K., Mod.Phys.Lett.A, 26 (2011) 893.

[19] McGaugh S. S., Phys.Rev.Lett., 95 (2005) 171302.

[20] Nesbet R. K., IBM J.Res.Develop., 42 (1998) 53.

[21] Mannheim P. D., ApJ, 479 (1997) 659.

[22] Mannheim P. D. and O'Brien J. G., Phys.Rev.Lett., 106 (2011) 121101.

[23] Mannheim P. D. and O'Brien J. G., Phys.Rev.D, 85 (2012) 124020.

[24] O'Brien J. G. and Mannheim P. D., MNRAS, 421 (2012) 1273

[25] McGaugh S. S., Phys.Rev.Lett., 106 (2011) 121303.

[26] Famaey B. and McGaugh S. S., Living Rev. Relativity, 15 (2012) 10.

[27] Moffat J. W., JCAP, 2006 (2006) 4.

[28] Moffat J. W. and Brownstein J. R., ApJ, 636 ((2006) 721.

[29] Nesbet R. K., arXiv:1004.5097v2, (2010).

[30] Komatsu E. et al, ApJS, 180 (2009) 330.

[31] Komatsu E. et al, ApJS, 192 (2011) 18.

[32] Mannheim P. D. and Kazanas D., ApJ, 342 (1989) 635.

[33] Riess A. G. et al, ApJ, 116 (1998) 1009.

[34] Perlmutter S. et al, ApJ, 517 (1999) 565.

[35] Weinberg S., Gravitation and Cosmology: Principles and Applications of the General Theory of Relativity (Wiley, NY) 1972.

[36] Nesbet R. K., Variational Principles and Methods in Theoretical Physics and Chemistry (Cambridge Univ. Press, NY) 2003. 